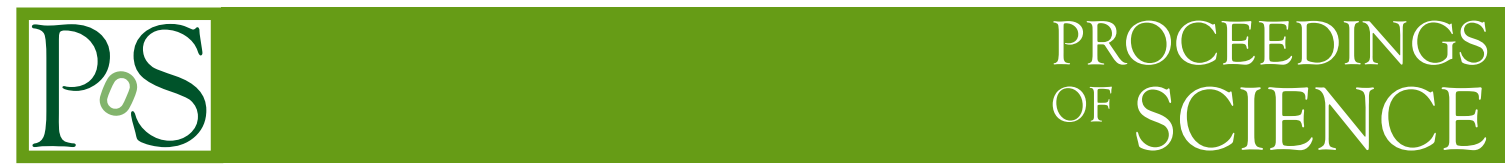

\title{
CMS Pixel Operations and Performance
}

\author{
Karl M. Ecklund ${ }^{* \dagger}$ \\ Rice University \\ E-mail: Karl.Ecklund@rice.edu
}

The CMS Pixel Detector has been commissioned in beam collisions at the LHC. This contribution describes the commissioning and operational experience with first LHC beams. Results discussed include the charge measurement calibration, timing alignment, hardware efficiency, thresholds, and beam backgrounds. The present status of the detector and operational performance during the first LHC run are presented.

19th International Workshop on Vertex Detectors

June 6 - 112010

Loch Lomond, Scotland, UK

\footnotetext{
*Speaker.

On behalf of the CMS Collaboration.
} 

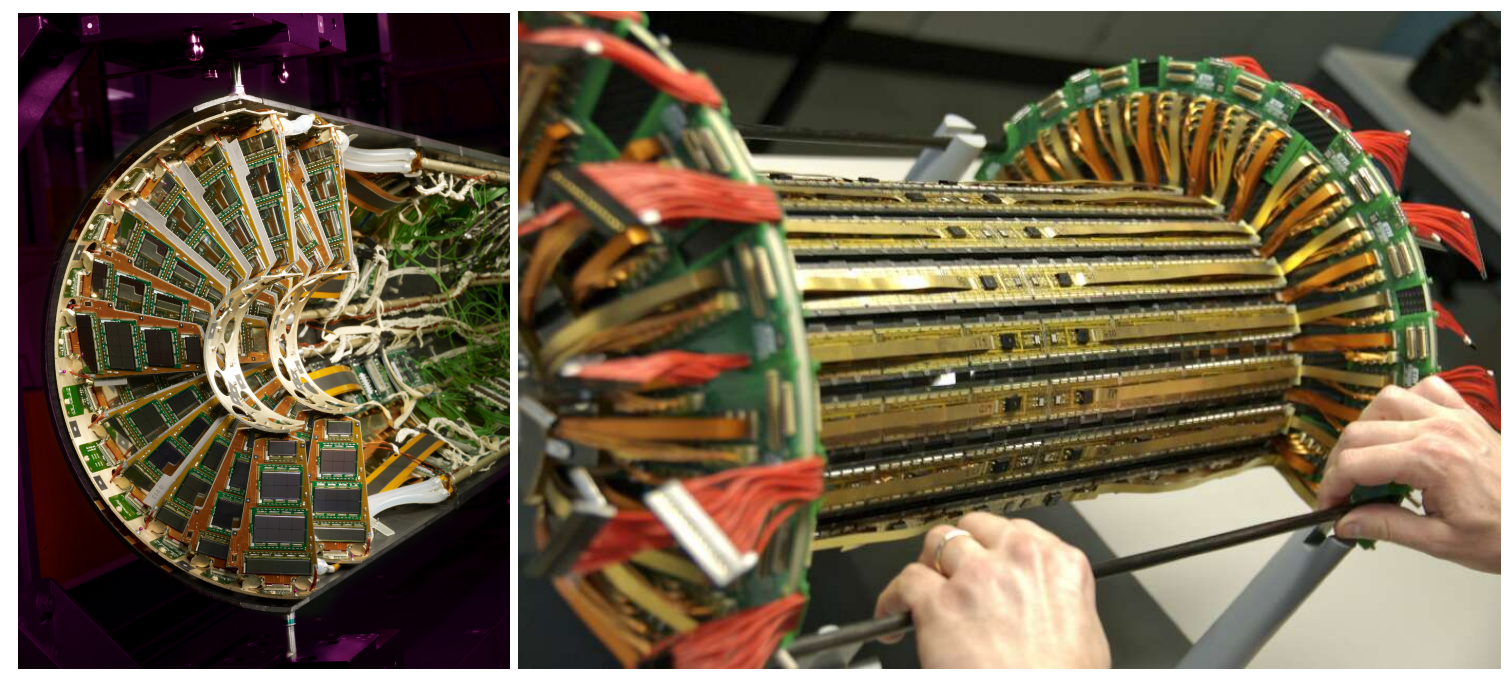

Figure 1: Photo of endcap (one of four) and barrel (one of two) pixel assemblies.

\section{CMS Pixel Detector}

The CMS Pixel detector sits at the center of the Compact Muon Solenoid (CMS) experiment [1] at CERN's Large Hardon Collider (LHC), which is designed to provide $10^{34} \mathrm{~cm}^{-2} \mathrm{~s}^{-1}$ luminosity of $14 \mathrm{TeV}$ proton-proton collisions and $10^{27} \mathrm{~cm}^{-2} \mathrm{~s}^{-1}$ luminosity in $5.5 \mathrm{TeV} \mathrm{Au}-\mathrm{Au}$ collisions. Initial operations have included collisions at much lower luminosities ${ }^{1}$ during commissioning of the $\mathrm{LHC}$ in proton-proton collisions at $900 \mathrm{GeV}, 2.36 \mathrm{TeV}$, and $7 \mathrm{TeV}$. The silicon pixel detector is designed [2] to provide high-efficiency, low-occupancy tracking and vertexing information in the high multiplicity collisions provided by the LHC. Rectangular "n-on-n" silicon sensors of $\approx 270$ $\mu \mathrm{m}$ thickness have surface metalization for $100 \mu \mathrm{m}$ by $150 \mu \mathrm{m}$ pixels. The sensors are arranged into three faceted barrel layers concentric with the beam pipe at radii of 4.3, 7.2, and $11 \mathrm{~cm}$ from the beam axis and two endcap disks on each end of the barrel providing three-hit coverage to psuedorapidities $(\eta)$ of 2.1 , and two-hit coverage to $\eta=2.5$. The barrel layers are assembled from rectangular sensors bump bonded to read-out chips and attached to power, control, and readout cables; each assembly is referred to as a module. The disks each have 24 trapezoidal double-sided "blades" holding seven rectangular sensors of varying sizes and using the same read-out chip as the barrel. Each side of the blade forms an independent module for readout. Figure 1 shows completed half shells of one endcap and barrel assembly, which close around the beam pipe. The total area of the detector is just under $1 \mathrm{~m}^{2}$, with 66 million pixels. The pixels are connected to a custom ASIC [3] that performs amplification, shaping, and discrimination with pixel-by-pixel adjustable threshold for a zero-suppressed read out of hit pixels' charge measurement. A data acquistion and control system [4] collects a sparse data stream from the read-out chips and provides trigger, clock, and control signals, all via optical fiber.

\footnotetext{
${ }^{1} \mathrm{~A}$ few $10^{29} \mathrm{~cm}^{-2} \mathrm{~s}^{-1}$ was acheived in late May 2010. In the same units, the LHC goal for 2010 is $10^{32}$.
} 


\section{Commissioning and Calibration}

The pixel read-out and control system was initially commisioned in 2008 after installation in August. A quick checkout (3-4 days) verified optical communication to all detector modules. This was followed by adjustment of the front end electronics parameters. The read-out chip has 25 DAC settings and a few registers. Many of the chip settings could be taken from test-stand optimization during module construction, but 10 of the 25 settings were re-optimized in situ including those controlling voltage regulators, pulse shaping, address encoding, timing, and thresholds. Many of these have temperature sensitivity requiring re-optimization. The signals from the read-out chips are transmitted by optical fiber to front-end driver (FED) boards where flash ADCs receive the data packets from each optical link. Parameters of the FED are adjusted to correctly decode the data received. The retuning of these read-out chip and FED parameters took 2-3 weeks, dominated by the threshold adjustment. The initial commissioning and front-end electronics calibration in 2008 are described in some detail in references [5] and [6].

In 2009 leading up to beam collisions at the LHC, further optimization of the front-end electronics parameters was made. The coolant temperature was dropped by $7^{\circ} \mathrm{C}$ to $7.4^{\circ} \mathrm{C}$, requiring a minor reoptimization of the read-out-chip DAC set points. The entire process took only a few days and was easily completed as all of CMS was restarting operations in advance of the beam collisions.

During this period further tuning of the thresholds was also possible. Using charge-injection calibration runs, the thresholds are measured by varying the VCAL DAC that controls the injected charge and measuring the efficiency versus injected charge. The VCAL DAC was cross calibrated using x-ray sources during barrel module construction and testing, giving an average charge calibration of 65.5 electrons/count. The RMS over readout chips of the conversion factor is 8.9 electrons/count, consistent with expected variations of capacitors in the readout chip fabrication process. The spread includes variation of injection capacitors for each pixel cell of the chip. At present, a common conversion function is applied to all chips and pixels: $Q=65.5 * V C A L-414$ electrons. These variations of $15-20 \%$ in the charge-injection circuit dominate the systematic uncertainty on the absolute charge scale. Chip-by-chip and even pixel-by-pixel calibrations will be possible in the future using in situ high-statistics collision data.

The resulting "s-curve" (figure 2) from a charge-injection scan is fit to an error function. The threshold is defined at $50 \%$ efficiency, while the slope at the threshold is a measure of the instrinsic noise of the discriminator circuit. By iteratively adjusting the threshold DACs on each read-out chip and repeating the threshold measurement, it was possible to reduce the thresholds to an average of 2500 electrons. The limiting factor in the threshold tuning is cross talk: signals from firing pixels feed into the input of other pixel amplifiers causing them to also register a hit. The thresholds were lowered in steps until cross talk was encountered, then raised slightly into a stable region. The intrinsic noise of the discriminator inferred from the slope of the turn-on region has a mean of 85 electrons with a RMS of 26 electrons for the endcap sensors, and a mean of 140 electrons and RMS of 35 electrons for the barrel sensors. The difference in noise is very likely due to the larger capacitance of the barrel sensor design, though this has not been studied in detail.

Figure 2 shows the distribution of thresholds used for beam collision running in 2009-2010. The readout-chip average threshold is determined from s-curve scans on a subset of pixels for 

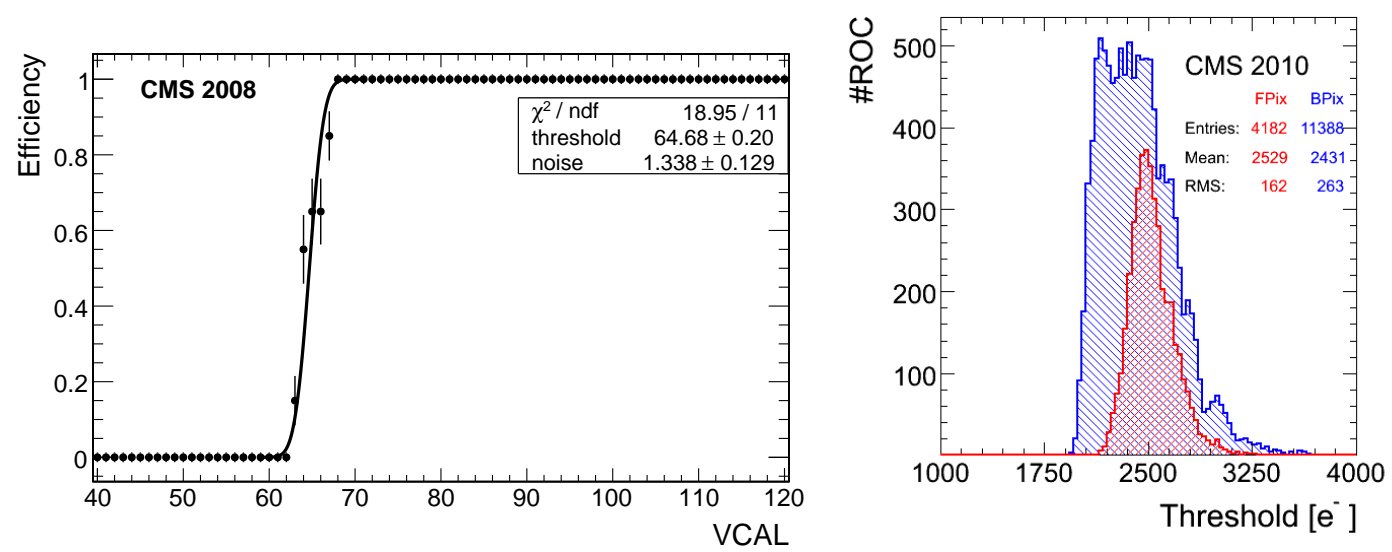

Figure 2: S-curve from a threshold scan on a single pixel. The distribution of the average thresholds per readout-chip. One VCAL count corresponds to 65.5 electrons.

each read-out chip. Threshold variations within a chip are small (100-200 electrons) thanks to threshold trim bits adjusted to minimize the spread during pre-installation testing. The overall average threshold is 2500 electrons, and represents the so-called "absolute threshold" for a hit to be recorded by the read-out chip. The "in-time threshold" adds the requirement that the hit be recorded in the correct $25 \mathrm{~ns}$ LHC beam crossing. Due to the finite slewing rate of the front-end amplifier, smaller pulse height signals go over threshold later. The in-time threshold is approximately 750 electrons larger than the absolute threshold.

Charge injection is also used to calibrate the ADC to charge conversion for pixel hits. For each pixel, a linear function is fit to the average ADC response as a function of injected charge. For the front-end electronics parameters in use for beam collisions in 2009-2010, the distribution of linear fit parameters is given in figure 3. The fits are done in units of VCAL, which is converted to electrons using the average calibration obtained using x-ray sources during module testing. The RMS spread of the gain (inverse slope) is about 15\% of the mean gain, and the RMS for the pedestal (offset) is about $32 \%$ of the mean pedestal.

\section{Beam Operations}

CMS entered beam collision running with $98.8 \%$ of the barrel and $96.4 \%$ of the endcap pixels operational. In the barrel, the non-functional pixels come from modules with broken wirebonds or failed readout electronics, present since installation time [6,5]. In the endcap, there are seven bad modules ( $0.5 \%$ each). Five modules have slow analog output and share a common analog optical hybrid. The precise cause for this is not understood, and these failures developed early in 2009 during commissioning. One module has a single bad read-out chip; the remaining 20 can be recovered with software changes. An additional module has excessive noise on the analog optical output, which is likely to be from dirt on an optical connection. The disabled endcap pixel modules can be further diagnosed and potentially repaired during a shutdown of a least three months. The design of CMS allows a "fast" extraction and insertion of the pixels. In 2009, repairs were made to 

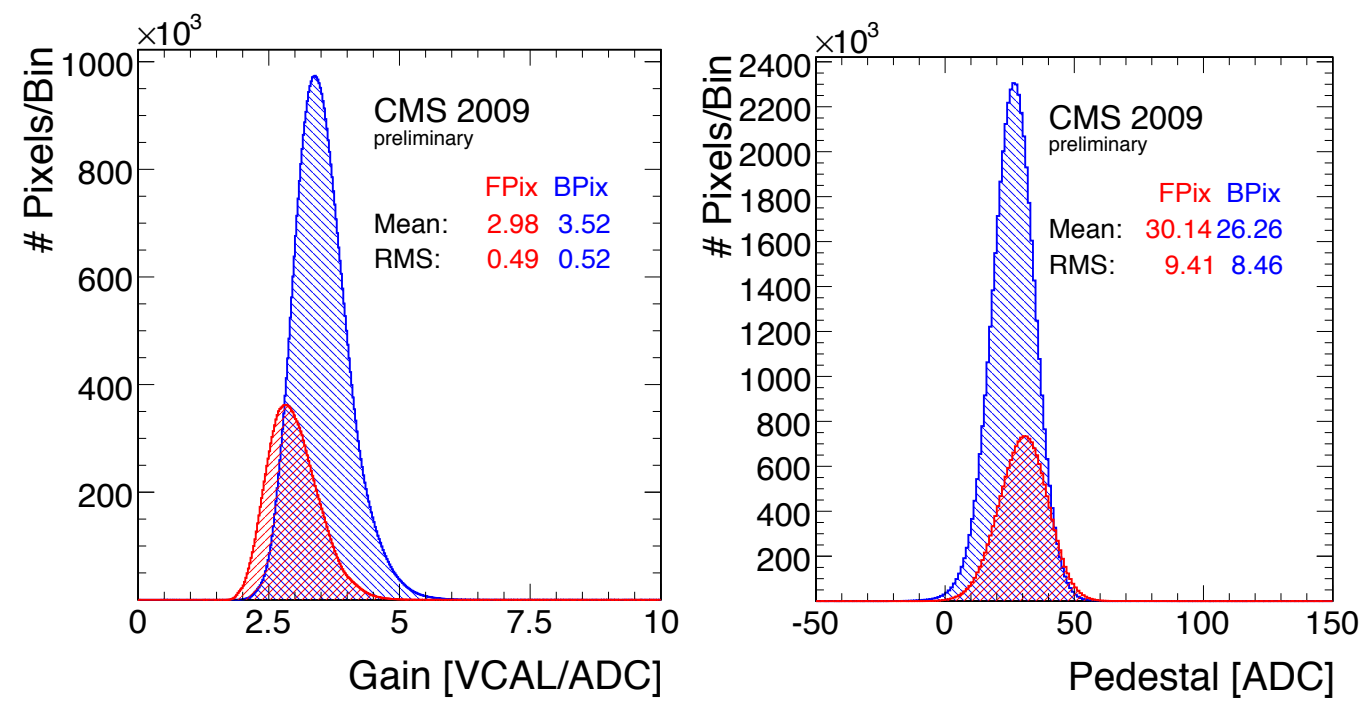

Figure 3: Distribution of linear charge calibration parameters, gain and pedestal, defined as the ADC value for zero injected charge.
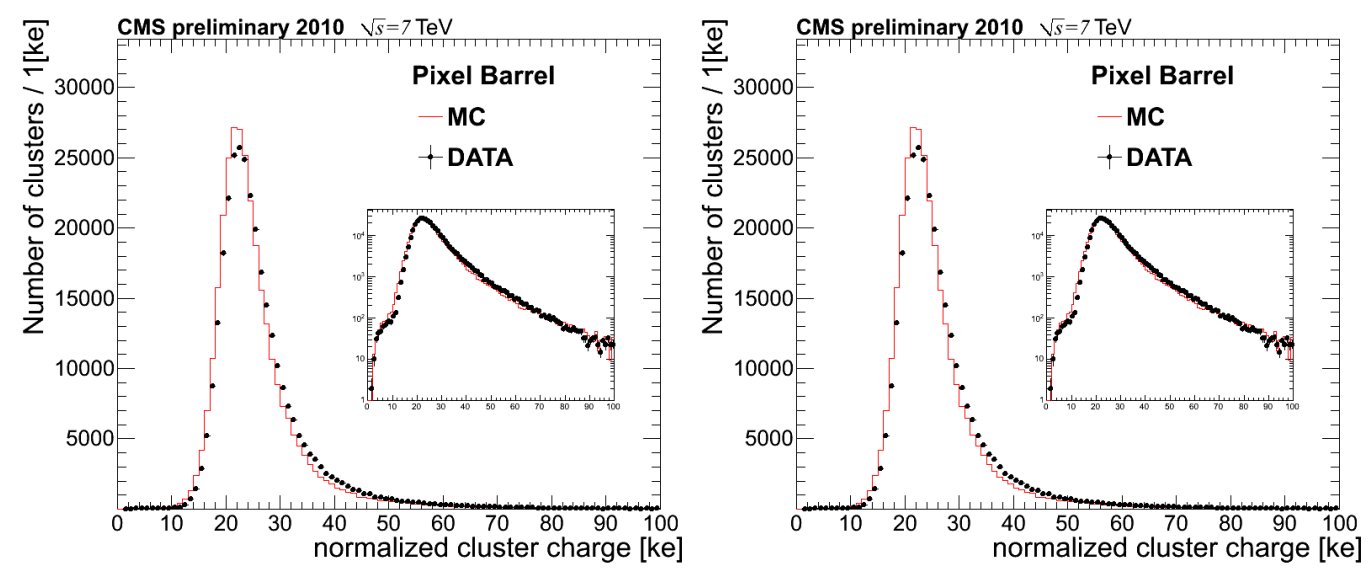

Figure 4: Cluster charge distribution corrected to normal incidence.

the endcap pixels, including replacement of a panel with a broken wire bond. Sufficient spare parts are available for repair in 2012 after the initial LHC run.

The LHC delivered $\sqrt{s}=0.9 \mathrm{TeV}$ and 2.36 TeV proton-proton collisions late in 2009, followed by $\sqrt{s}=7 \mathrm{TeV}$ collisions since the end of March 2010. Early during this period the pixel detector was commissioned for beam operations. Although comsic ray runs were used for initial commissioning [6], the rates are extremely low compared to those in the LHC environment, and the arrival of cosmic ray tracks is not synchronous with the LHC beam crossing clock, leading to complexities of timing adjustment and performance measurement.

Using the charge calibration obtained from charge injection normalized with test stand $\mathrm{x}$-ray measurements, the following charge distributions are measured in $7 \mathrm{TeV}$ proton-proton collisions. The cluster charge distributions for hits assigned to tracks with $p_{T}>2 \mathrm{GeV} / c$ are corrected for the impact angle to normal incidence on the sensor. A miniumum ionizing particle is expected to 

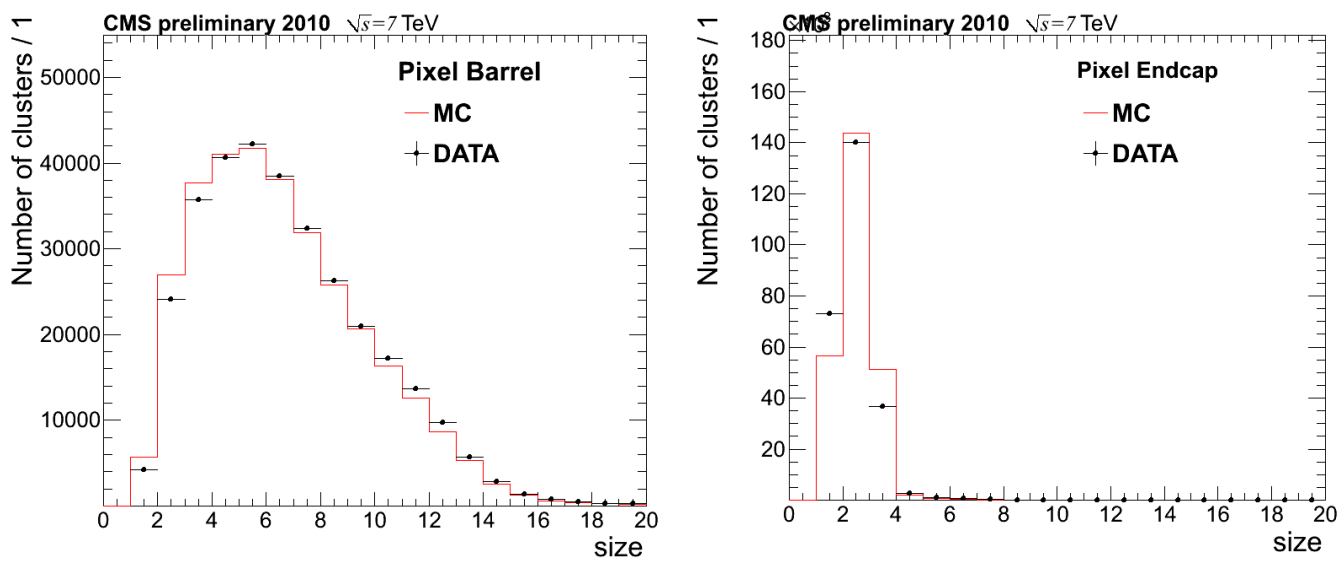

Figure 5: Cluster size distribution.
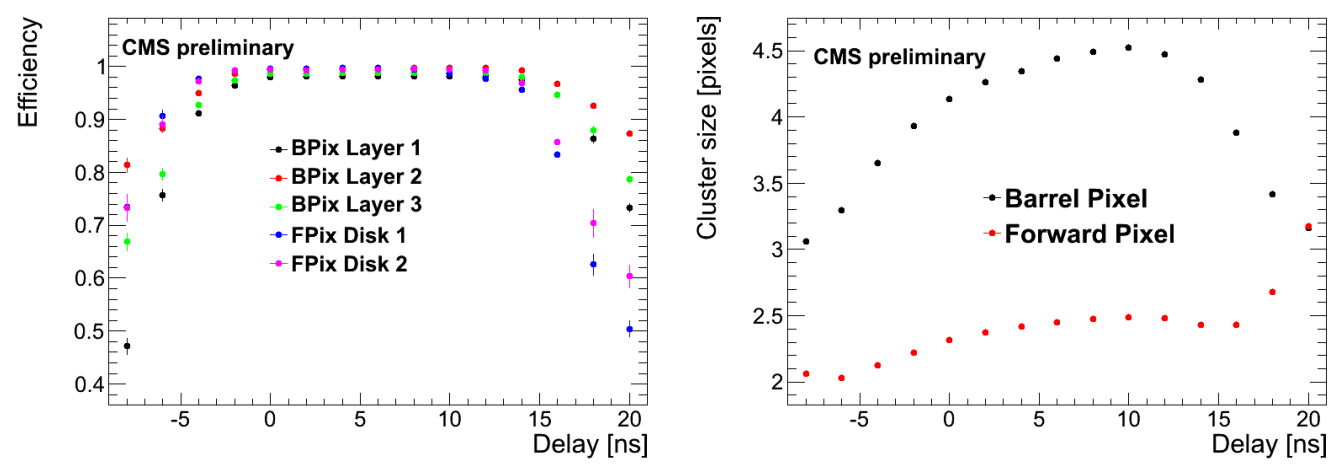

Figure 6: Variation of hit efficiency (left) and mean cluster size (right) versus clock delay.

deposit 22000 electrons in the pixel sensors. Figure 4 shows peaking at the expected charge deposition and a good agreement with simulation for the shape and normalization. The peak positions are correct to $4 \%$ and $2 \%$ for the endcap and barrel regions, respectively. The widths are 10-15\% wider than simulation, but in remarkable agreement this early in an experiment. The cluster size distributions (fig. 5) also show good agreement in the barrel and less so in the endcap, which is dominated by smaller cluster sizes by the geometry. Further improvements are expected as the simulation is tuned to more accurately reflect the electronics performance for the chosen operating parameters, including thresholds and non-linearities.

The read-out chip runs at the LHC crossing frequency of $40 \mathrm{MHz}$ and is sensitive by design to a single beam crossing. Hit pixels are associated with a particular beam crossing. Proper timing adjustment is even finer than the 25 ns clock period, because of the time-walk effect, which causes small pulse height hits to fire the discriminator at later times than large pulse height due to the risetime of signals. The optimum delay of the LHC clock will record large pulses just after the clock transition, allowing the maximum time for small pulses to go over threshold. That is, we adjust the clock delay to minimize the in-time threshold.

The delays of all clock distribution circuits in the pixels were scanned in 2 ns steps during one of the first collision runs. The efficiency has a wide plateau in the delay scan as expected (fig. 6), 


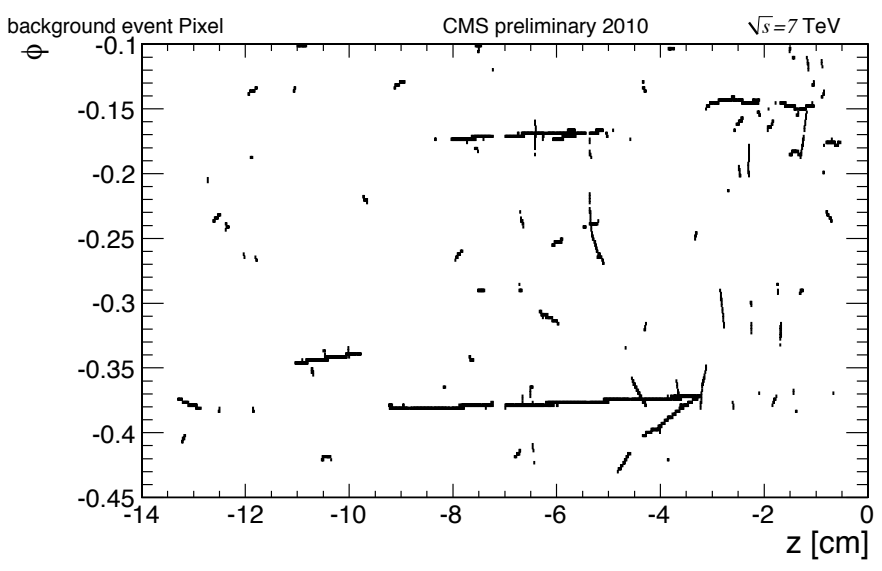

Figure 7: Zoomed-in hit map on barrel ladder for beam-background event.

because clusters are not lost until no hit pixel is recorded. To confirm the largest delay with high efficiency as the optimum delay, the mean cluster charge and mean cluster size is also plotted versus the delay in fig. 6 . With the optimum delay setting, small charge pixels are also recorded in the same bunch crossing as the large charge pixels, giving a maximum cluster charge. Using the scan, a delay of +10 ns was chosen as the optimum, while for some early runs a delay of +6 ns was used. (The zero delay setpoint is completely arbitrary.) With the same scan data, the relative alignment of each clock distribution circuit was adjusted to better than $2 \mathrm{~ns}$.

One feature of early collision running was somewhat surprising. Events with very large pixel occupancies appeared, and it became clear these came from beam backgrounds. Figure 7 shows a hit map for a single pixel ladder of the barrel of one such event. It is consistent with a shower of particles traveling nearly parallel to the beam axis at a small radius. The high occupancy on a given event is only appears on a few modules out of about 1000. The pixel occupancy distribution in fig. 8 for a zero bias trigger shows a strong peak as expected from miniumum bias protonproton collisions, but also a very long tail from beam-related backgrounds. These are consistent with beam-gas interactions within approximately $100 \mathrm{~m}$ of the interaction point. Hella Snoek will report in detail later in this conference [7] on studies of the beam backgrounds.

When such events are present and triggered on, they can cause some difficulty for the pixel data acquisition system. The large number of hits on a few sensors will cause those read-out chips to transmit large data packets. While the receiving front-end driver electronics (FED) truncates large events and thus can continue with additional triggers, the affected channel is inefficient on subsequent triggers until there is sufficient time for the backlog of data to be transmitted. To mitigate these effects, the firmware of the FED module was modified to automatically recover channels affected by large occupancy. The improved firmware counts the number of triggers missed due to the large data packet and temporarily removes the affected channel from the data stream until the front end has "caught up" with the triggers again. This firmware change is an example of a feature added to the data acquisition system to cope with actual running conditions at the LHC in a first-generation pixel silicon detector. We expect to continue to evolve the data acquisition and online software system to deal with similar features as the luminosity and instantaneous rates increase. 


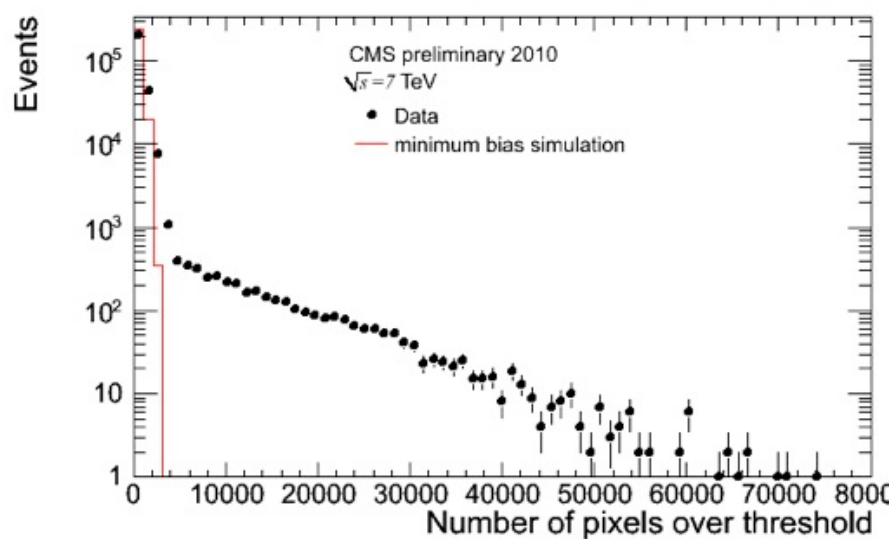

Figure 8: Hit occupancy for zero bias triggers showing two components: low occupancy consistent with minimum bias simulation and high occupancy tail consistent with beam gas collisions.

\section{Performance}

The pixel detector performance has been measured using the early collision data. The hit resolution can be measured using tracks which intersect the edges of two overlapping modules. The geometry of the barrel is such that the overlap is always in the azimuthal coordinate. The same technique was used with cosmic rays earlier [6]. By using the difference of hit residuals between the two sensors, alignment, track extrapolation, and multiple scattering contributions cancel, leaving a measurement of the hit resolution. For pairs of modules with more than 30 shared tracks, resolutions of $\sigma_{u}=12.7 \pm 2.3 \mu \mathrm{m}$ (azimuthal coordinate) and $\sigma_{v}=28.2 \pm 1.9 \mu \mathrm{m}$ (along the beam direction) are measured [8]. These can be compared to expectations from a detailed sensor simulation of $\sigma_{u}=14.1 \pm 0.5 \mu \mathrm{m}$ and $\sigma_{v}=24.1 \pm 0.5 \mu \mathrm{m}$. Note that from detailed sensor simulations [9], we expect a larger resolution at the edges than on average in the azimuthal coordinate $\left(\sigma_{u} \approx 10 \mu \mathrm{m}\right)$, due to geometrical effects and the Lorentz angle.

With a demonstrated hit resolution consistent with expectations, it is interesting to examine performance in vertex position measurements [8]. Additional performance results will be covered by A. Venturi later in the conference [10]. The primary vertex position is found using prompt tracks in an adaptive algorithm. The primary vertex distribution for a single LHC fill is shown in fig. 9 . The distributions are dominated by the beam size, $0.25 \mathrm{~mm}$ in $x$ and $y$ transverse to the beam, and $39 \mathrm{~mm}$ in $z$. The primary vertex resolution is well modeled in simulation, and also studied in a two-vertex method. Tracks associated with the primary vertex are split into two subsets that are separately fit. The difference is used as an estimate of the resolution and compared to simulation in figure 10 as a function of the number of tracks associated with the vertex. The agreement is very respectable.

The performance for secondary vertexing from long-lived heavy flavor particles has also been investigated [11]. Using less than just $1 \mathrm{nb}^{-1}$ of $7 \mathrm{TeV}$ collision data, jets of $p_{T}>40 \mathrm{GeV} / \mathrm{c}$ in the central $|\eta|<1.5$ region were examined by a basic b-tagging algorithm. The three dimensional signed impact parameter to the primary vertex is shown in figure 11. The data is overlayed with expectations from a mix of simulated data from light jets and charm and bottom jets. A clear signal of long-lived hadrons is seen in the data, in nice agreement with simulation. Figure 11 also 

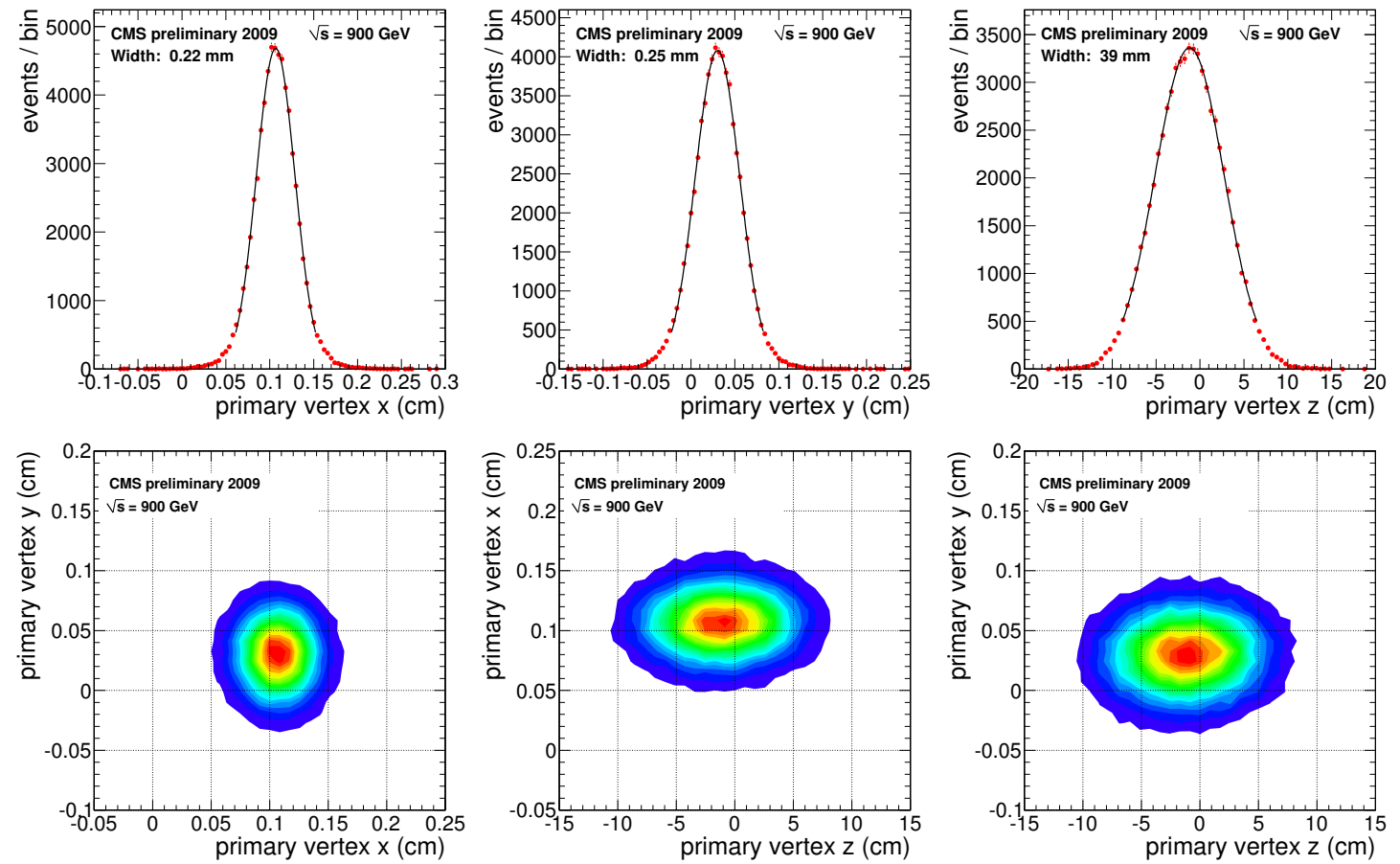

Figure 9: Reconstructed location of primary vertex in a single LHC fill.
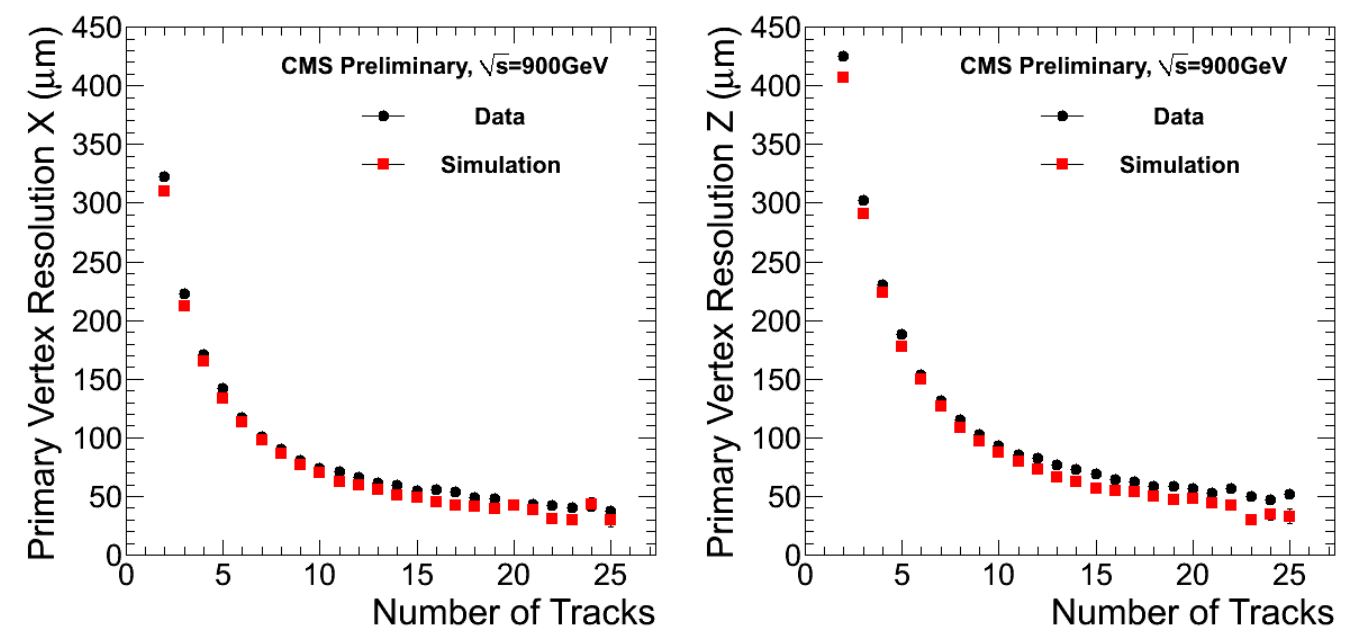

Figure 10: Primary vertex resolution as a function of number of tracks.

shows the significance of the impact parameter, demonstrating that clear secondary vertexes can be reconstructed using the pixel detector and used for heavy flavor tagging in physics analysis. At this early stage a number of cleanly separated secondary vertex dijet events have been observed.

\section{Summary}

The CMS pixel detector is operational with $98 \%$ of the channels working. It is performing 

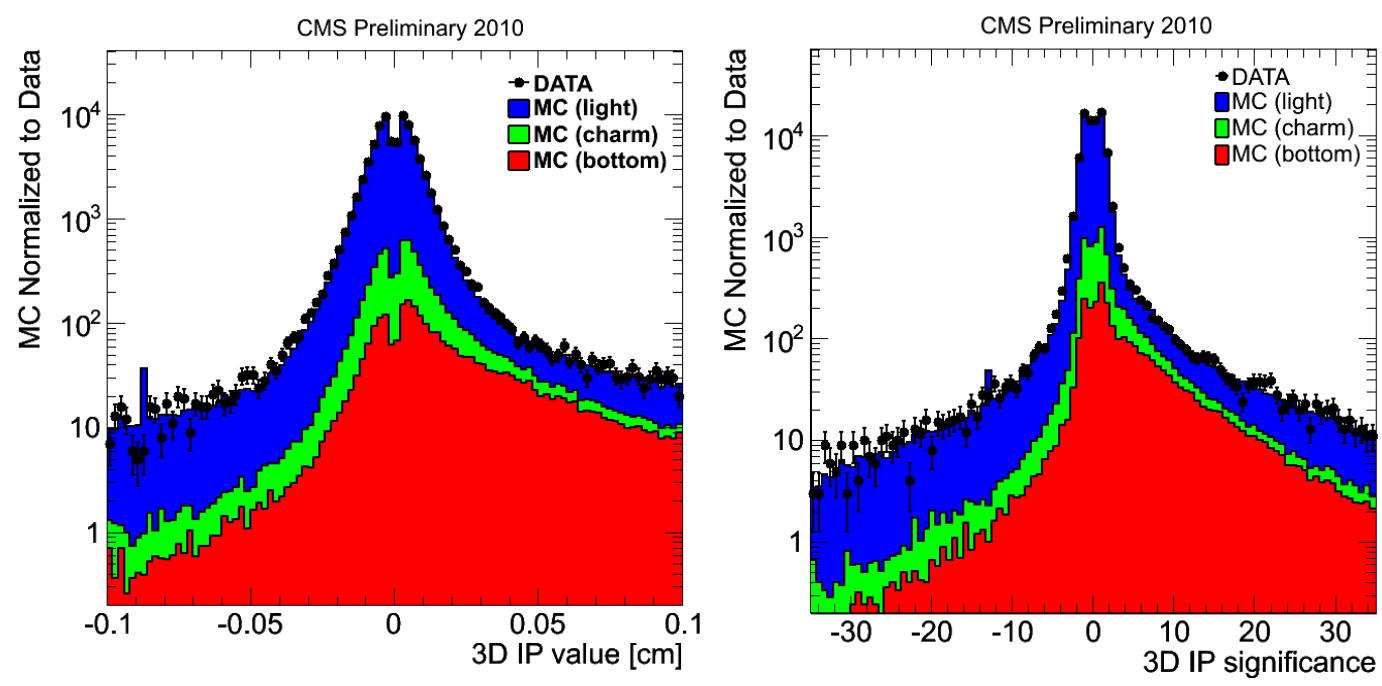

Figure 11: Three dimensional impact parameter and significance in early collision data, showing expected excess in positive flight direction from secondary vertexes in long-lived charm and bottom jets.

in LHC beam collisions very well after commisioning and timing adjustments. Pixel thresholds of 2500 electrons have been achieved, giving hit resolutions of 15-25 $\mu \mathrm{m}$ measured using the overlap technique, consistent with expectations for the resolution of the inclined tracks at the edge of overlapping modules. The pixel detector contributes crucially to reconstruction of primary interaction vertexes and secondary vertexes from heavy flavor, with early indications of excellent performance. Beam commissioning of the data acquisition system has successfully dealt with high occupancy events from beam-gas interactions and continues in the face of higher luminosity from the Large Hadron Collider. We look forward to many years of productive physics output from CMS and the pixel detector.

\section{References}

[1] CMS Collaboration, The CMS experiment at the CERN LHC, JINST 3 (2008) S08004.

[2] CMS Collaboration, The CMS tracker system project: technical design report, CMS TDR 5 (1998) and addendum (2000).

[3] H. Chr. Kästli et al., Design and performance of the CMS pixel detector readout chip Nucl. Instrum. Meth. A565 (2006) 188 [physics/0511166].

[4] D. Kotlinski, The design of the CMS pixel detector system, Nucl. Instrum. Meth. A477 (2002) 446.

[5] D. Kotlinski, Status of the CMS pixel detector in proceedings of Pixel 2008, JINST 4 (2009) P03019.

[6] CMS Collaboration, Commissioning and Performance of the CMS Pixel Tracker with Cosmic Rays, JINST 5 (2010) T03007 [arXiv: 0911.5434$].$

[7] H. Snoek, Beam Background Effects in the CMS Pixel Detector in proceedings of Vertex 2010, PoS (Vertex2010).

[8] CMS Collaboration, Tracking and Vertexing Results from First Collisions, CMS PAS TRK-10-001. 
[9] M. Swartz et al., Observation, modeling, and temperature dependence of doubly peaked electric fields in irradiated silicon pixel sensors, Nucl. Instrum. Meth. A565 (2006) 212.

[10] A. Venturi, CMS Alignment, Tracking and Physics Performance Results, in proceedings of Vertex 2010, PoS (Vertex2010).

[11] CMS Collaboration, B-tagging observables and event display from first $7 \mathrm{TeV}$ data, CMS DPS-2010/015. 